\title{
Microbiological characteristics of perianal streptococcal dermatitis: a retrospective study of 105 patients in a 10-year period
}

\author{
Anja Šterbenc ${ }^{1}$, Katja Seme ${ }^{1}$, Liza Lea Lah², Olga Točkova ${ }^{3}$, Tina Kamhi Trop ${ }^{4}$, Nataša Švent-Kučina ${ }^{1}$, Mateja Pirš ${ }^{1 凶}$
}

\begin{abstract}
Beta-hemolytic streptococci (BHS) are the most common causative agents of perianal streptococcal dermatitis (PSD). This study evaluates the distribution of BHS isolates in perianal bacterial cultures. We retrospectively reviewed microbiological results for perianal BHS that were isolated in our laboratory between 2006 and 2015 . We identified a total of 105 BHS isolates from rectal swabs and swabs of clinically intact perianal skin. The majority of BHS were of group A (GABHS) $(73 / 105 ; 69.5 \%)$, followed by group B BHS (GBBHS) $(27 / 105 ; 25.7 \%)$, and non-group A or B BHS (5/105; 4.8\%). The distribution of GABHS was age-specific, with the majority of GABHS obtained from young children. All BHS isolates were susceptible to penicillin. GABHS were universally susceptible to clindamycin, whereas $1.4 \%$ were resistant to erythromycin. GBBHS were resistant to erythromycin and clindamycin in $14.8 \%$ and $7.4 \%$ of cases. In addition, we wanted to emphasize the importance of correct diagnosis of PSD. Hence, we provide a review of protocols that can decrease the time to diagnosis and treatment of PSD, reduce patients' discomfort, and prevent unnecessary diagnostic procedures.
\end{abstract}

Keywords: beta-hemolytic streptococci, perianal swab samples, culture, streptococcal dermatitis

Received: 23 November 2016 | Returned for modification: 26 November 2016 | Accepted: 30 November 2016

\section{Introduction}

Beta-hemolytic streptococci (BHS) are well-known causative agents of cutaneous, oropharyngeal, and invasive infections (1). Perianal streptococcal dermatitis (PSD) typically affects children 6 months to 10 years old and is usually caused by group A BHS (GABHS) with the species name Streptococcus pyogenes $(2,3)$. In contrast, PSD has rarely been reported in adults $(4,5)$, where group B BHS (GBBHS) with the species name Streptococcus agalactiae are regarded as the most common causative agents (6). In rare cases, BHS of groups C (GCBHS) and G (GGBHS) as well as Staphylococcus aureus can also cause perianal disease $(6,7)$. PSD can be diagnosed relatively easily if physicians are familiar with the classical presentation of the disease, which includes perianal erythema, edema, and itching together with rectal pain and blood-streaked stools $(2,6,8)$. Infants typically also present with episodes of intermittent irritability (9). Diagnosis can be confirmed using swabs of the perianal area for bacterial culture; another possibility in some cases is the use of rapid antigen detection tests (RADTs) (10, 11). Initiation of an appropriate antibiotic treatment rapidly and drastically improves patients' symptoms. However, treatment is often delayed because differential diagnosis of PSD includes a variety of clinical conditions (e.g., irritant diaper dermatitis, candidiasis, infection with Enterobius vermicularis, inverse psoriasis, seborrheic dermatitis, chronic inflammatory bowel disease, histiocytosis, zinc deficiency, and, rarely, sexual abuse) $(2,8,12)$. In addition to oral penicillin and amoxicillin, PSD can also be treated with clindamycin, erythromycin, clarithromycin, or cefuroxime $(1,6,13,14)$.

This study evaluates the distribution of BHS isolates from rectal and perianal skin swab samples and provides a review of protocols that could potentially decrease the time to diagnosis and treatment of PSD, reduce patients' discomfort, and prevent un- necessary diagnostic procedures.

\section{Materials and methods}

We retrospectively reviewed microbiology laboratory records and searched for BHS isolates in the perianal area. BHS isolates from rectal or perianal skin swabs that were submitted to the Institute of Microbiology and Immunology, Faculty of Medicine, University of Ljubljana, Slovenia, between January 2006 and December 2015 were included in the study. Only the first BHS isolate from the perianal region of each patient was included in the study. Sampling sites included the rectum, perianal skin, and perineum. For each patient with a positive BHS culture, data were collected on patient age and sex, streptococcal species, and antimicrobial susceptibility. Identification was confirmed to the species level by colony morphology, catalase test, and a commercial latex agglutination test (PathoDxtra Strep Grouping Kit, Thermo Fisher Scientific, Waltham, MA, USA) or matrix-assisted laser desorption ionization-time of flight (MALDI-TOF) mass spectrometry (Bruker Daltonics, Bremen, Germany). Antimicrobial susceptibility was determined using the disk diffusion method according to the Clinical Laboratory Standards Institute (CLSI) guidelines until April 2014 and afterwards the European Committee on Antimicrobial Susceptibility Testing (EUCAST) guidelines $(15,16)$.

\section{Results}

In the 10-year study period, we identified a total of 105 BHS isolates isolated from the rectum or perianal skin region of the same number of patients. GABHS, GBBHS, and non-group A or B BHS were cultured in a total of 73/105 (69.5\%), 27/105 (25.7\%), and 5/105 (4.8\%) cases, respectively (Fig. 1). The distribution of GABHS, GBBHS, and non-group A or B BHS according to age groups

${ }^{1}$ Institute of Microbiology and Immunology, Faculty of Medicine, University of Ljubljana, Ljubljana, Slovenia. ${ }^{2}$ Department of Infectious Diseases, Ljubljana University Medical Center, Ljubljana, Slovenia. ${ }^{3}$ Department of Dermatology, Ljubljana University Medical Center, Ljubljana, Slovenia. ${ }^{4}$ Department of Gastroenterology, Hepatology, and Nutrition, Children’s Hospital, Ljubljana University Medical Center, Ljubljana, Slovenia. $\bowtie$ Corresponding author:mateja.pirs@mf.uni-lj.si 
is presented in Figure 2. The majority of streptococcal isolates were obtained from children younger than 15 (89/105; 84.7\%). The median age of patients was 5 (average 11.8 years, age range 1 to 84 years) and 73 out of 105 (69.5\%) were male (Fig. 3).

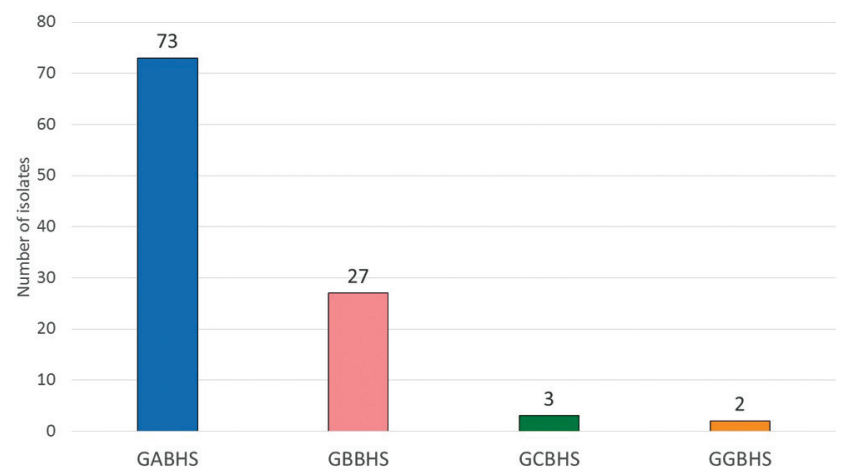

Figure 1 | Proportion of BHS in perianal isolates. GABHS = group A beta-hemolytic streptococci. GBBHS = group B beta-hemolytic streptococci. GCBHS = group $\mathrm{C}$ beta-hemolytic streptococci. GGBHS = group $\mathrm{G}$ beta-hemolytic streptococci.

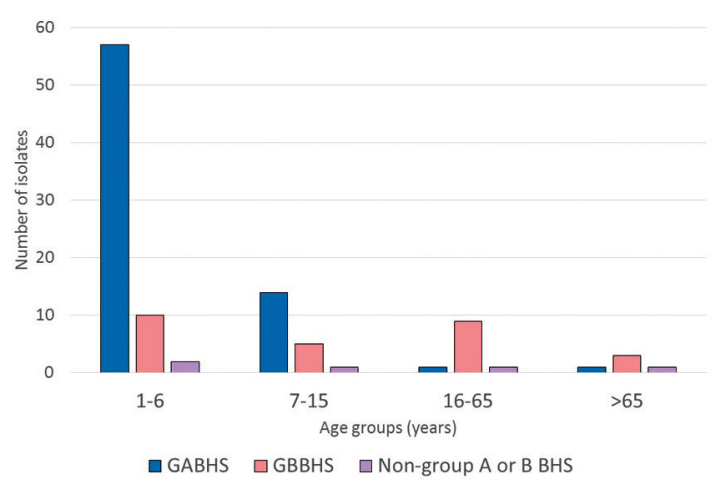

Figure 2 | Age-related distribution of a total of 105 streptococcal perianal isolates. GABHS = group A beta-hemolytic streptococci. GBBHS = group B betahemolytic streptococci. Non-group A or B BHS = non-group A or B beta-hemolytic streptococci (groups C and G).

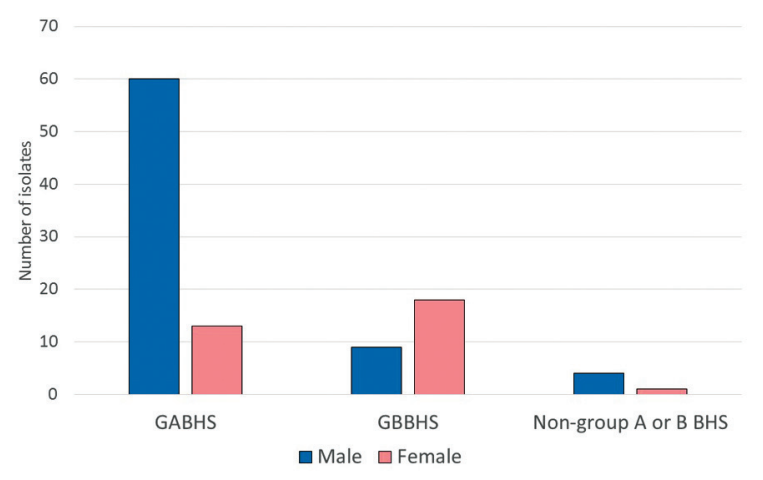

Figure 3 | Distribution of a total of 105 streptococcal perianal isolates according to the patient's sex. GABHS = group A beta-hemolytic streptococci. GBBHS = group $B$ beta-hemolytic streptococci. Non-group $A$ or $B$ BHS $=$ non-group $A$ or $B$ beta-hemolytic streptococci (groups C and G).

The seasonal distribution of all GABHS, GBBHS, and non-group A or B BHS perianal cultures is presented in Figure 4. Almost half $(46.6 \%)$ of the GABHS perianal cultures were obtained during the spring (between March and June), and the second peak was observed in December. Similar to GABHS, the number of GBBHS perianal cultures peaked in the spring and winter months. Only a small number of non-group A or B BHS belonging to groups $\mathrm{C}$ and $\mathrm{G}$ were isolated. All GABHS isolates were susceptible to penicillin and clindamycin, whereas the rate of erythromycin resistance was $1.4 \%$. All GBBHS isolates were susceptible to penicillin, whereas $14.8 \%$ and $7.4 \%$ were resistant to erythromycin and clindamycin, respectively. The number of non-group A or B BHS isolates was too low to reliably assess antimicrobial susceptibility; nonethe-

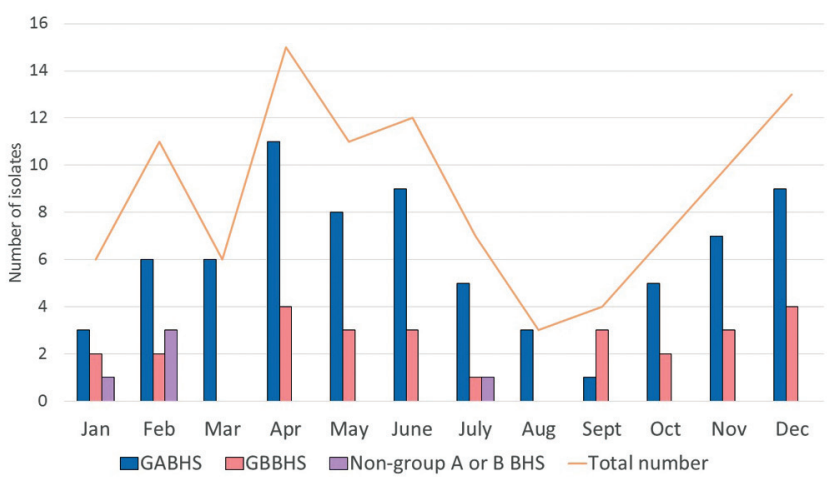

Figure 4 | Seasonal distribution of a total of 105 streptococcal perianal isolates. $\mathrm{GABHS}=$ group $\mathrm{A}$ beta-hemolytic streptococci. GBBHS = group B beta-hemolytic streptococci. Non-group A or B BHS = non-group A or B beta-hemolytic streptococci (groups $C$ and G).

less, all of the isolates were susceptible to penicillin.

\section{Discussion}

Although perianal dermatitis caused by infection with BHS is a well-described clinical entity in children, its incidence is most likely significantly underestimated in both children and adults due to frequent misdiagnosis (6). This study emphasizes the importance of correct and rapid diagnosis of PSD.

PSD classically presents as a well-demarcated perianal erythema with or without exudate, which may centrifugally spread to the penis or vulva and is present in more than $90 \%$ of patients with PSD (1, 2, 17-19). It is often accompanied by edema, infiltration, and tenderness (3). According to the literature $(2,3,17-20)$, patients' signs and symptoms include perianal itching (78-100\%), pain on defecation (52\%), constipation (47\%), blood-streaked stools (20-35\%), and anal fissures (26\%). PSD mostly occurs in children 6 months to 10 years old and is more common in boys $(2,3,20)$. Differential diagnosis of PSD is vast and, unfortunately, patients are commonly overlooked $(2,8,12)$. Patients can present with symptoms that have lasted for several years and may have even undergone several unnecessary diagnostic procedures, such as colonoscopy or rectoscopy $(3,8,12)$. Inappropriate treatments with topical antifungal agents and steroids or oral preparations for pinworms obscure the typical clinical presentation of PSD and worsen its symptoms (8). Due to prolonged and inappropriately treated or untreated PSD, patients may develop anal fissures, which can result in painful defecation, leading to constipation and toilet avoidance $(11,19)$.

Our study evaluated a 10-year distribution of BHS in swabs from the rectum and perianal skin that were submitted to our laboratory for bacterial culture. As shown in Figure 1, the majority of isolates were GABHS, followed by GBBHS and non-group A or B BHS (groups C and G). BHS were isolated from perianal cultures obtained from patients of all age groups except for children younger than 1; however, we observed significant differences in species distribution (Fig. 2). In preschool and primary school age groups, the majority of perianal streptococcal cultures grew GABHS, whereas GBBHS were the most commonly isolated BHS in adults. Non-group A or B BHS isolates obtained from swabs of clinically intact perianal skin were rare. Based on our results, we can assume that GABHS represents the most probable cause of PSD in children, whereas GBBHS is the presumed causative agent of PSD in adults. As shown in Figure 2, more than $84 \%$ of perianal 
streptococcal cultures were obtained from children under 15. Our results are in accordance with previous studies, suggesting that, although PSD predominantly occurs in young children, it should not be considered an exclusively pediatric disease $(1,3,6)$. More than $69 \%$ of BHS perianal isolates obtained in our study were from male patients, which is in agreement with previous observations suggesting male predilection of PSD (Fig. 3) (2, 3, 20).

Seasonal distribution of BHS isolates observed in our study showed remarkable consistency with previous reports of PSD in children $(3,17)$. Interestingly, seasonal distribution of PSD cases exhibits a characteristic pattern of pharyngeal GABHS infections in temperate climates and supports the idea of autoinoculation through digital contamination or ingestion of GABHS $(3,17)$. In our study, we were not able to assess the proportion of concurrent pharyngeal GABHS carriage, but it has previously been shown that up to $92 \%$ of individuals with PSD test positive for pharyngeal GABHS (17).

Although appropriate sampling is crucial for laboratory confirmation of etiology in PSD, there are currently no clear recommendations regarding the adequacy of different clinical samples used in diagnosing PSD. The affected area(s) should be cleaned with saline and thoroughly swabbed. Anal, perianal, and perineal swabs represent preferred clinical samples, whereas stool samples are not recommended. Needle aspiration of a leading edge of the inflamed area can also be used; however, the low sensitivity for detecting causative agents and its relative invasiveness limit its role in routine practice $(21,22)$. Processing of swab samples obtained for RADT must be performed in accordance with the manufacturer's instructions. Swab samples obtained for bacterial culture should be placed in a transport medium (e.g., Stuart's or Amies) and sent at room temperature to the microbiology laboratory as soon as possible. Standard laboratory procedure is cultivation of BHS on blood agar (21). As emphasized by some authors, high clinical suspicion of PSD should encourage physicians to specifically ask for BHS culture because stool culture might fail to detect BHS $(3,12,20)$. In addition, cultivation allows isolation and identification of all BHS as well as $S$. aureus, which is also a possible etiologic agent.

Alternatively, GABHS- and GBBHS-RADTs are sometimes used as a point-of-care test; however, GBBHS-RADTs should be avoided due to their low sensitivity and specificity (23). Unfortunately, only a few studies have evaluated the clinical sensitivity and specificity of RADTs for detecting extrapharyngeal GABHS infection (2, $10,11)$. Depending on the RADT used, the sensitivity for extrapharyngeal GABHS ranged from $77.9 \%$ to $98.0 \%$, suggesting that these tests may represent a rapid, practical, and accurate alternative diagnostic tool for point-of-care differentiation of GABHSassociated PSD from other conditions with similar presentations (e.g., irritant dermatitis, candidiasis, and pinworm infestation) (10). Nevertheless, physicians should be aware of the age-specific distribution of GABHS and GBBHS infection and must account for these differences when deciding to use GABHS-RADT for screening PSD. One of the major caveats of using GABHS-RADT in diagnosing GABHS-associated PSD is the lack of formal approval for extrapharyngeal testing $(11,12)$. In addition, a negative GABHSRADT result warrants additional testing by conventional bacterial culture especially in adults, where PSD is most commonly caused by GBBHS $(1,6,10)$. A subset of perianal dermatitis cases can also be caused by non-group A or B BHS (groups C and G), as well as $S$. aureus $(6,7)$. Thus, in children, GABHS-RADT can be used as a point-of-care test, whereas RADTs are not recommended in adults. Cultivation of BHS is the preferred microbiological method for diagnosing PSD in adults and in children with perianal dermatitis and a negative GABHS-RADT result.

Early initiation of antibiotic treatment provides rapid improvement of symptoms (8). Our study has shown that susceptibility of BHS to penicillin remains excellent. GABHS isolates are rarely resistant to erythromycin or clindamycin, whereas higher resistant rates for both antibiotics were observed in GBBHS isolates. A 7- to 10-day course of oral penicillin V (50,000 to 100,000 IU/ $\mathrm{kg}$ ) is considered to be the initial treatment of choice for pediatric GABHS-associated PSD (2, 3, 13, 14, 24, 25). However, recurrence of the disease may occur in up to $39 \%$ of children treated and a repeated course of antibiotics is necessary, whereas some advocate prolonged treatment (e.g., 14-21 days) (26-30). Unfortunately, studies comparing the optimal duration of antibiotic therapy are lacking. Alternatively, children can be treated with oral amoxicillin (50 mg/ $\mathrm{kg} /$ day) and, if compliance is an issue, one dose of penicillin G 1.2 M IU im can be used in children weighing $>27 \mathrm{~kg}$, and one dose of penicillin G 600,000 IU im in children weighing < $27 \mathrm{~kg}(24,25)$. In children with penicillin allergy, midecamycin (40 $\mathrm{mg} / \mathrm{kg} /$ day), clarithromycin ( $15 \mathrm{mg} / \mathrm{kg} /$ day), or clindamycin (30 $\mathrm{mg} / \mathrm{kg} /$ day) can be used $(24,25)$, although data regarding their efficacy rely solely on a subset of treated children $(2,4,14,20,31)$. To date, cefuroxime is the only alternative antibiotic in treatment of PSD that has been assessed in a randomized controlled trial (13). In comparison to penicillin, an increased efficacy of a 7-day course of cefuroxime ( $20 \mathrm{mg} / \mathrm{kg} /$ day) was observed, with shorter duration of symptoms and faster bacterial eradication $(13,14)$. However, the study was not blinded and, because no follow-up was performed after the end of the treatment, optimal duration of antibiotic therapy with cefuroxime could not be evaluated (13). Furthermore, usage of cephalosporins is not recommended for treatment of BHS due to their broad spectrum of activity, which can lead to the development of antibiotic resistance in other bacteria (24). In addition to oral therapy, patients can also receive topical treatment with antiseptics (e.g., chlorhexidine) or antibiotics (e.g., bacitracin, mupirocin, fusidic acid, erythromycin, and gentamicin), although their usefulness remains uncertain (2, $3,14,19,30)$. Unfortunately, no controlled trials were conducted to evaluate the efficacy of antimicrobial therapy for non-GABHSassociated PSD. In adults with predominantly GBBHS-induced PSD, a 7- to 10-day treatment with oral penicillin V (1-1.5 M IU/ day) is considered standard therapy $(1,6,24,25)$. Alternatively, patients can receive one dose of penicillin G $1.2 \mathrm{M} \mathrm{IU/day} \mathrm{im} \mathrm{or,}$ when penicillin allergy is suspected, oral midecamycin (400 mg tid), clarithromycin (250-500 mg bid), and azithromycin (500 mg 1st day, $250 \mathrm{mg}$ 2nd-5th day) (25). However, physicians should be aware of important differences between pediatric and adult cases of PSD. Only $42 \%$ of adult patients with PSD are successfully treated with the first course of oral antibiotics, possibly due to the higher minimal inhibitory concentration for penicillin in GBBHS compared to GABHS, hence a higher dosage of the same antibiotic might be necessary, whereas some advocate prolonged treatment $(6,32)$. Kahlke et al. (6) have clearly shown that the presence of concomitant dermatological and/or anorectal conditions that have not yet developed in children (e.g., hemorrhoids, skin tags, anogenital warts, and anal cancer) contribute to reduced rates of successfully treated infections in adults $(1,6)$. In addition, these conditions can present with symptoms that are otherwise observed in PSD (e.g., perianal erythema in patients with hemorrhoids) and may be the reason for frequent misdiagnosis in adults 
(6). Although complications of PSD are rare, a urine analysis may be performed to screen for possible post-streptococcal glomerulonephritis $(19,30)$.

In both children and adults, follow-up is crucial due to frequent relapses of the disease (8). Repeated antibiotic treatment of these cases is usually successful $(3,8)$. Short-term recurrence of PSD might be caused by poor compliance with the antibiotic therapy, inappropriate dosage, and intra-familial or close contact transmissions, especially in children (2). Thus, screening and eventual treatment of symptomatic family members of patients with recurrent PSD may be warranted (12). A change of personal hygiene tools (e.g., toothbrush and towels) should be recommended after completion of antibiotic treatment. Patients should be advised not to share personal hygiene items with family members that could be BHS carriers. Simple measures such as thorough handwashing can be effective in preventing further infections (24).

Our study is based solely upon a retrospective review of laboratory records with BHS isolates from rectal and perianal skin swab samples and presumed diagnosis of perianal streptococcal infections, which is its main limitation. No data on clinical presentation and diagnosis of PSD, antibiotic treatment, and potential relapse(s) or the presence of concomitant diseases were collected. Further studies with clinically and microbiologically confirmed cases of PSD are needed to confirm our observations.

To conclude, we observed seasonal and age-specific distribution of GABHS, GBBHS, and non-group A or B BHS in rectal and perianal skin bacterial cultures. Thus, symptoms that include perianal itching, rectal pain, and blood-streaked stools, as well as bright red, well-demarcated perianal erythema with edema, infiltration, and tenderness on a clinical examination of a preschool child are highly suspicious of PSD caused by GABHS. Based on our data, a subset of PSD cases can also be diagnosed in adulthood, where GBBHS are the most likely causative agents. Anal, perianal, or perineal swabs are preferred clinical samples for microbiological confirmation of diagnosis of PSD, whereas stool samples are not recommended. GABHS-RADTs enable rapid diagnosis especially in children; however, a negative result warrants further testing with cultivation of BHS. In adult patients, cultivation of BHS is always necessary due to the poor performance of GBBHS-RADTs. Swab samples of perianal lesions obtained for conventional bacterial culture are the most reliable diagnostic tool for diagnosing perianal dermatitis because they also enable detection of less common causative agents. As shown in our study, BHS are universally susceptible to penicillin and, because symptoms improve dramatically with appropriate antibiotic therapy, treatment with oral penicillin should not be delayed.

\section{References}

1. Zhang C, Haber RM. The ABCs of Perineal Streptococcal Dermatitis: Case Series and Review of the Literature. J Cutan Med Surg. 2016; [Epub ahead of print].

2. Kokx NP, Comstock JA, Facklam RR. Streptococcal perianal disease in children. Pediatrics. 1987;80:659-63

3. Jongen J, Eberstein A, Peleikis HG, Kahlke V, Herbst RA. Perianal streptococcal dermatitis: an important differential diagnosis in pediatric patients. Dis Colon Rectum. 2008;51:584-7.

4. Neri I, Bardazzi F, Marzaduri S, Patrizi A. Perianal streptococcal dermatitis in adults. Br J Dermatol. 1996;135:796-8.

5. Bafounta ML, Bloch P, Kernbaum S, Saiag P. Group A beta-hemolytic Streptococcus: an unusual etiology of perianal dermatitis in an adult? Ann Dermatol Venerol. 1998;125:902-4.

6. Kahlke V, Jongen J, Peleikis HG, Herbst RA. Perianal streptococcal dermatitis in adults: its association with pruritic anorectal diseases is mainly caused by group B Streptococci. Colorectal Dis. 2013;15:602-7.

7. Heath C, Desai N, Silverberg NB. Recent microbiological shifts in perianal bacterial dermatitis: Staphylococcus aureus predominance. Pediatr Dermatol. 2009;26:696-700.

8. Brilliant LC. Perianal streptococcal dermatitis. Am Fam Physician. 2000;61:391-7.

9. Shouval DS, Schurr D, Nussinovitch M. Presentation of perianal group A streptococcal infection as irritability among children. Pediatr Dermatol. 2008;25:568-70.

10. Clegg HW, Dallas SD, Roddey OF, Martin ES, Swetenburg RL, Koonce EW, et al. Extrapharyngeal group A Streptococcus infection: diagnostic accuracy and utility of rapid antigen testing. Pediatr Infect Dis J. 2003;22:726-31.

11. Cohen R, Levy C, Bonacorsi S, Wollner A, Koskas M, Jung C, et al. Diagnostic accuracy of clinical symptoms and rapid diagnostic test in group A streptococcal perianal infections in children. Clin Infect Dis. 2015;60:267-70.

12. Block SL. Perianal dermatitis: much more than just a diaper rash. Pediatr Ann. 2013;42:12-4.

13. Meury SN, Erb T, Schaad UB, Heininger U. Randomized, comparative efficacy trial of oral penicillin versus cefuroxime for perianal streptococcal dermatitis in children. J Pediatr. 2008;153:799-802.

14. Olson D, Edmonson MB. Outcomes in children treated for perineal group A betahemolytic streptococcal dermatitis. Pediatr Infect Dis J. 2011;30:933-6.

15. Clinical and Laboratory Standards Institute. (M100-S24). Performance Standards for Antimicrobial Susceptibility Testing - Twenty- Fourth Informational Supplement, vol. 34. Wayne, PA: Natl Comm Clin Lab Stand; 2014.

16. The European Committee on Antimicrobial Susceptibility Testing. Breakpoint tables for interpretation of MICs and zone diameters. Version 5.0, 2015. [cited 2016 Nov 06]. Available from: http://www.eucast.org.
17. Mogielnicki NP, Schwartzman JD, Elliott JA. Perineal group A streptococcal disease in a pediatric practice. Pediatrics. 2000;106:276-81.

18. Echeverría Fernández M, López-Menchero Oliva JC, Marañón Pardillo R, Míguez Navarro C, Sánchez Sánchez C, Vázquez López P. [Isolation of group A betahemolytic Streptococcus in children with perianal dermatitis]. An Pediatr (Barc). 2006;64:153-7.

19. Lehman R, Pinder S. Streptococcal perianal infection in children. BMJ. 2009;338: b1517.

20. Wright JE, Butt HL. Perianal infection with beta haemolytic streptococcus. Arch Dis Child. 1994:70:145-6.

21. Garcia LS, Isenberg HD. Clinical Microbiology Procedures Handbook. 3rd ed. Washington, DC: ASM Press; 2010.

22. Piso RJ, Pop R, Wieland M, Griesshammer I, Urfer M, Schibli U, et al. Low sensitivity of needle aspiration cultures in patients with cellulitis/erysipelas. Springerplus. 2016;5:1578.

23. Donders GG, Vereecken A, Salembier G, Spitz B. Accuracy of rapid antigen detection test for group B streptococci in the indigenous vaginal bacterial flora. Arch Gynecol Obstet. 1999;263:34-6.

24. Tomažič J, Strle F, s sod. Infekcijske bolezni. Ljubljana: Združenje za infektologijo, Slovensko zdravniško društvo; 2014/2015. Poglavje 6, Okužbe kože in mehkih tkiv; p. 152-62.

25. Gilbert DN, Moellering RC Jr, Eliopoulos GM, Chambers HF, Saag MS. The Sanford Guide to Antimicrobial Therapy 2010. 40th ed. Sperryville, VA: Antimicrobial Therapy, Inc.; 2010.

26. Marks VJ, Maksimak M. Perianal streptococcal cellulitis. J Am Acad Dermatol 1988;18:587-826.

27. Duhra P, Ilchyshyn A. Perianal streptococcal cellulitis with penile involvement. Br J Dermatol. 1990;123:793-6.

28. Teillac-Hamel D, de Prost Y. Perianal streptococcal dermatitis in children. Eur Dermatol 1992;2:71-4.

29. Morgenroth HHA. Perianale Streptokokkeninfektionen, eine mögliche Ursache für den Pruritus ani, perianale Dermatitis, Defäkationsschmerzen, Stuhl-verhalt und blutige Stuhlauflagerungen. Monatsschr Kinderheilkd; 1995. 144, p. 55-58.

30. Herbst R. Perineal streptococcal dermatitis/disease: recognition and management. Am J Clin Dermatol. 2003;4:555-60.

31. Rehder PA, Eliezer ET, Lane AT. Perianal Cellulitis. Cutaneous Group A Streptococcal Disease. Arch Dermatol. 1988; 124:702-4.

32. Farley MM. Group B streptococcal disease in nonpregnant adults. Clin Infect Dis. 2001;33:556-61. 